\title{
Hyper Spectral Image Classification using Multi Labelled, Multi-Scale and Multi-Angle CNN with MS-MA BT Algorithm
}

\author{
Sujata Alegavi , Raghavendra Sedamkar
}

\begin{abstract}
For classifying the hyperspectral image (HSI), convolution neural networks are used widely as it gives high performance and better results. For stronger prediction this paper presents new structure that benefit from both MS - MA BT (multi-scale multi-angle breaking ties) and CNN algorithm. We build a new MS - MA BT and CNN architecture. It obtains multiple characteristics from the raw image as an input. This algorithm generates relevant feature maps which are fed into concatenating layer to form combined feature map. The obtained mixed feature map is then placed into the subsequent stages to estimate the final results for each hyperspectral pixel. Not only does the suggested technique benefit from improved extraction of characteristics from CNNs and MS-MA BT, but it also allows complete combined use of visual and temporal data. The performance of the suggested technique is evaluated using SAR data sets, and the results indicate that the MS-MA BT-based multi-functional training algorithm considerably increases identification precision. Recently, convolution neural networks have proved outstanding efficiency on multiple visual activities, including the ranking of common two-dimensional pictures. In this paper, the MS-MA BT multi-scale multi-angle CNN algorithm is used to identify hyperspectral images explicitly in the visual domain. Experimental outcomes based on several SAR image data sets show that the suggested technique can attain greater classification efficiency than some traditional techniques, such as support vector machines and conventional deep learning techniques.
\end{abstract}

Index Terms: Convolution neural networks (CNNs), MS-MA BT (multi-scale multi-angle breaking ties), Hyperspectral image (HSI), Classification, Synthetic aperture radar (SAR)

\section{INTRODUCTION}

Hyperspectral image (HSI) is commonly used in the remote sensing world to take full benefit of the structure of hundreds of discrete streams over a given image. Hyperspectral image needs precise and robust identification methods to obtain the characteristics from the image. Due to the complicated design of the image scene hyperspectral image has been regarded as a particularly difficult issue (i.e., combined pixels, big amounts of information and restricted practice tests), and therefore many attempts have been made to address this problem in the last few decades. In the initial phase of HSI evaluation, spatial domain classifiers such as

Revised Manuscript Received on July 22, 2019

Sujata Alegavi , Raghavendra Sedamkar $\mathrm{PhD}$. Research Scholar, Dean Academic

Department of Electronics \& Telecommunication and Computer Engineering, Thakur College of Engineering \& Technology,

Mumbai, India support vector machines (SVM) [2], MS-MA BT (multiscale multi-angle breaking ties) have produce a better achievement in understanding the image scenes. Recent technological progress offers more successful solutions to the ranking of HSI. These techniques aim to distinguish HSI using both spectral and temporal data.

For example, a combined sparse model mixes the data from a few nearby pixels of the test pixel, which is shown to be an efficient way of improving classified efficiency.

Recently, in the field of computer vision, deep learning is of interest to researchers. Specifically, MS - MA BT and convolution neural network (CNN) [21][22] have attracted a lot of attention due to their exceptional results in many fields, such as Synthetic aperture radar [21], image detection [12]. In addition to removal of features, CNNs can discover depictions of features through several convolution sections. MS MA -BT classify in resolution and angles after concatenation feature. Unlike traditional rule-based removal techniques, CNNs can automatically discover characteristics from the initial pictures. In addition, MS-MA BT can be intended as an end-to-end algorithm that can immediately generate classification maps. In particular, multiplicative noise occurs in SAR images like speckle noise. It gives big impact on the training model of deep learning. It is therefore essential to extract the noise in our segmentation model. In addition, the SAR picture contains rich texture structures that are created by distinct land cover such as river, urban area and small objects. The input image patch for the deep model should contain meaningful structures instead of simple pixels. If the quantity of the patch is too small, the patch would not contain image buildings. It means that the picture patch design will not be depicted in the ultimate taught characteristics. If the patch volume is too large, the entry loop will contain redundant buildings. It will improve the network's stress and spend much more time in training and screening.

Many CNN designs and MS-MA BT algorithm have Therefore been introduced to HSI ranking. Usually, neighboring pixels have the same background characteristics in remote sensing pictures. Spectral-spatial multi-functions can be used to efficiently enhance ranking accuracy [5]. This result paper presents a multi-scale MS-MA BT CNN algorithm for semantic segmentation of the SAR picture. The model involves the phase of noise suppression, the phase of concatenation and the 


\section{Hyper spectral image classification using Multi Labelled, Multi-Scale and Multi-Angle CNN with MS-MA BT Algorithm}

the phase of ranking.

This paper is arranged as follows. The typical MSMA BT multi-scale CNN algorithm and the associated training process are described in Section 2. In Section 3, we experimentally evaluate the efficiency of our technique with different parameters. Finally, we will conclude by summarizing our outcomes in Section 4.

This result paper presents a multi-scale multi-angle MS-MA BT CNN algorithm for semantic segmentation of the SAR picture. The system involves the stage of noise suppression, the phase of concatenation function and the phase of ranking in Fig.1.In the noise removal stage, we use the sparse representation technique and design the reconstruction loss feature to obtain the cleared SAR image.

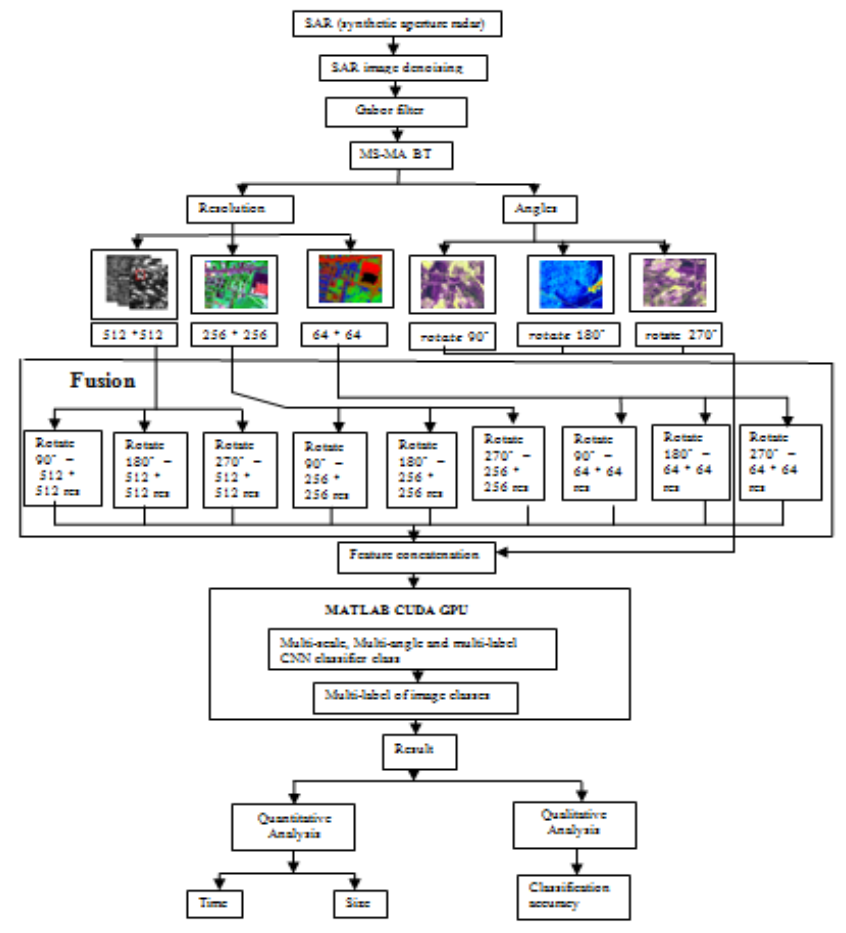

Fig 1: Overview of proposed approach

Our proposed system uses MSMA technique for segmenting the image which is called as multi scale \& multi angle. It gives results in resolution as well as angle. The BT algorithm has obtained excellent practical outcome as shown in MS-BT results in resolution and angles [20]. MS-MA BT provides resolution 512* 512 , resolution $256 * 256$ and resolution $64 * 64$. It rotates the picture into 90, 180, 270 and provides the highest outcome. All resolution results are conducted in fusion. Fusion provides a combination of angle and resolution. The concatenation layer of the function is used to link the characteristics with different scales, angle and depths. Then the multi-scale multi-angle convolution sections are used to know the multi-scale deep characteristics. Finally, the Multi-scale and multi-angle CNN classifier is used to acquire the SAR/ Hyperspectral image marks. The following observations are given in this paper: (1) We suggest a multi-scale CNN model and MS-MA BT algorithm with noise extraction
Convolution phase, concatenation phase and identification phase. This model takes into consideration the multiplicative noise and multi-scale, multi- angle features of the SAR images[21] (2) We decompose the multiplicative noise model into an additional model for extracting the noise. We are using sparse image loss function.(3) Segmentation precision is enhanced in our proposed method compared to state-of - the-art techniques. It results in quantitative and qualitative analysis. Quantitative analysis provides the outcome of time and size whereas the classification accuracy is given by qualitative analysis.

\section{MS-MA CNNALgORITHM}

In this result paper we are using MS-MA CNN algorithm.MS-MA CNN gives best result in resolution and angles.

\section{Algorithm: MS-MA CNN Algorithm}

Algorithm combines the BT strategy and MS cluster to select the most informative training samples. In order to better represent the MS-MA CNN algorithm, we need to define several variables. $q$ Demonstrates the number of samples to be marked for each iteration. We were using Algorithm to build a vector set X. We take one part of $\mathrm{X}$ as training set $\mathrm{XT}$ with $\mathrm{u}$ initial training samples and for validating the other part of set $X$, we take set $X V$ with I - $\mathrm{u}$ unlabeled samples. After the training set, XT, is labeled, it

is denoted as $B_{T}=\left\{\left(y i_{T,} x i_{Y}\right)\right\}_{T=1}^{u}$. When the validating set is unlabeled, $\mathrm{XV}=\left\{x i_{r}\right\}_{r=1}^{I-W}$, In the proposed algorithm, we select q new samples, such as $X_{M S-M A}=\left\{x i_{r}\right\}_{r=1}^{q}$ from the validated set, we label themas $B_{M S-M A}=\left\{y i_{r,} x i_{r}\right\}_{r=1}^{q}$ and then add $B_{M S-M A}$ into BT[21][22].

Input: Initial classifier parameter, $\omega$, training set, BT $=\left(y i_{r} x i_{F}\right)_{r=1}^{u}$, validating set, $X_{V}=\left\{X i_{r}\right\}_{r=1}^{I-U}$, mean-shift parameters, $\mathrm{h}$, candidate samples parameter, $\beta$ 1. Train the classifier with MS-MA CNN and achieve the parameter of the classifier $\omega$

2. Predict labels for XV using the classifier and obtain $\left(y i_{T}, x i_{r}\right)_{r=1}^{I-u}$

3. For each sample in the prediction $\left(y i_{r}, x i_{r}\right)_{r=1}^{I-u}$, compute its BT value as,

$\theta i_{Y}=\max p\left(y i_{r}=k \mid x i_{Y}, \omega\right)-\max p\left(y i_{r}=k \mid x i_{Y}, w\right)$.

4. Arrange $\{\theta\}_{r=1}^{I-u}$ in ascending order as $\left\{\theta_{z r}\right\}_{r=1}^{I-u}$, and based on the index $\left\{z_{\gamma}\right\}_{\gamma=1}^{I-U}$ take out the first $\beta$. q items from $\mathrm{XV}$ to be XBT $=\left\{X_{z Y}\right\}_{T=1}^{\beta-q}$.

5. Discover the q cluster centers for $X_{B T}=\left\{X_{E Y}\right\}_{T=1}^{\beta \times q}$ by mean shift. Select a sample closest to it for each cluster center and place all chosen q samples $\mathrm{q} X_{M S-M A}$.

$X_{M S-M A}=\left\{X i_{g}\right\}_{g=1}^{q}$ Label $X_{M S-M A}$ to obtain get

6. Update BT and XV.

$$
X_{M S-M A}=\left\{\left(y i_{g}, x i_{g}\right)\right\}_{g=1}^{q}
$$

$$
B_{T}=B_{T} \cup B_{M S-M A} ; X_{V}=X_{V}-X_{M S-M A}
$$

7. If a condition is not satisfied then return to step (1); else, stop the iteration. 
8. Initialize multi-scale $\mathrm{CNN}$ for semantic segmentation: set the size of the three scale and angle image patches as $\quad 512 \times 512,128 \times 128$ and $64 \times 64$ and $90^{\circ}, 180^{\circ}$ and $270^{\circ} \&$ the no., of the image classes is K;

9. Fusion is done for results obtained from resolution and angle. i.e., Combination of angle and resolution that is $90^{\circ}{ }_{*} 180^{\circ}{ }_{n} 270^{\circ}$ and $512 \times 512,128 \times 128,64 \times 64$ resolution;

10: Using a sparse depiction method, remove the SAR image speckle noise;
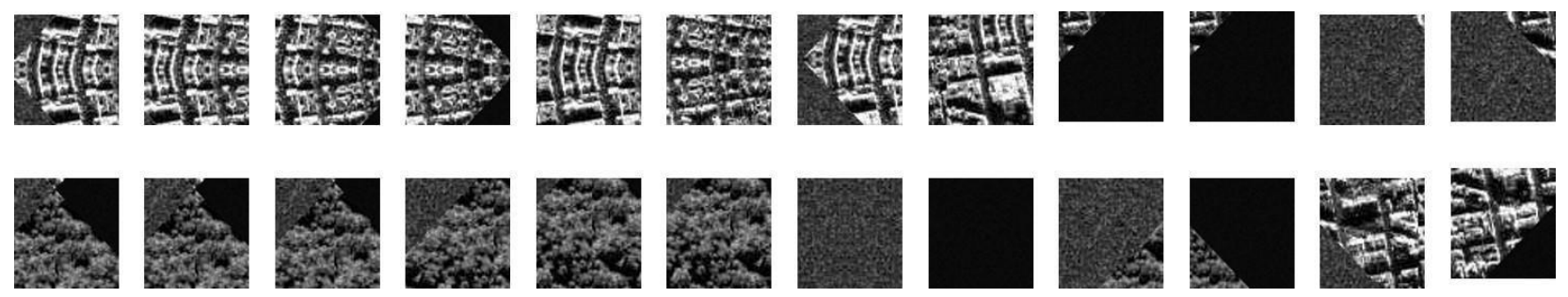

Fig. 2: Few Sample Images from our dataset. The data set contain 1049 samples.

In Table I We have taken dataset of size 1049 sample image with $60 \%$ training sample and $40 \%$ testing sample

Table I: Analysis of Angle, Resolution and Fusion of both.

\begin{tabular}{|c|c|c|}
\hline \\
\hline Dataset & S. No & Variant \\
\hline \multirow[t]{4}{*}{ Dataset A } & 1 & $0^{2}$ \\
\hline & 2 & $90^{\circ}$ \\
\hline & 3 & $180^{\circ}$ \\
\hline & 4 & $270^{2}$ \\
\hline \multirow[t]{3}{*}{ Dataset B } & 5 & $64 \times 64$ \\
\hline & 6 & $256 \times 256$ \\
\hline & 7 & $512 \times 512$ \\
\hline \multirow[t]{12}{*}{ Dataset C } & 8 & $0^{2}-64 \times 64$ \\
\hline & 9 & $0^{2}-256 \times 256$ \\
\hline & 10 & $0^{2}-512 \times 512$ \\
\hline & 11 & $90^{2}-64 \times 64$ \\
\hline & 12 & $90^{\circ}-256 \times 256$ \\
\hline & 13 & $90^{\circ}-512 \times 512$ \\
\hline & 14 & $180^{2}-64 \times 64$ \\
\hline & 15 & $180^{n}-256 \times 256$ \\
\hline & 16 & $180^{\circ}-512 \times 512$ \\
\hline & 17 & $270^{*}-64 \times 64$ \\
\hline & 18 & $270^{n}-256 \times 256$ \\
\hline & 19 & $270^{2}-512 \times 512$ \\
\hline
\end{tabular}

It should be observed that we use the image patches to represent the central pixel. We have to record the central pixel locations in the original SAR image. When we understand the image patch tag, the main pixel tag is acquired at the last phase of the network. All central pixel labels represent the SAR image semantic segmentation map.

\section{EXPERIMENTALRESULTS}

In our analysis, both qualitative and quantitative experiments were used to verify the effectiveness of the proposed multi-scale multi angle model of CNN. The main measures in our approach are shown in above algorithm. In this result paper, we are using the MS-MA BT multi-scale CNN model [21][22]. This chapter concentrates primarily on the texture image segmentation results and the actual SAR pictures.

\section{Implementation details:}

We solve the problem of semantic segmentation through a classification framework. We need to construct the data sets. For each pixel, we extract the image patch specifically. We randomly pick some patches as the training samples from the data collection. The rest of the data sets are considered as test samples. Detailed information about the data sets is provided in Table I.

TABLE II: Hyper-parameters of multi-scale CNN.

\begin{tabular}{|l|l|}
\hline Initial learning rate & $10^{-2}$ \\
\hline Epoch & 4 \\
\hline Frequency & 30 \\
\hline Fully connected layer & 10 \\
\hline
\end{tabular}

Furthermore, we contrast our strategy with angle,

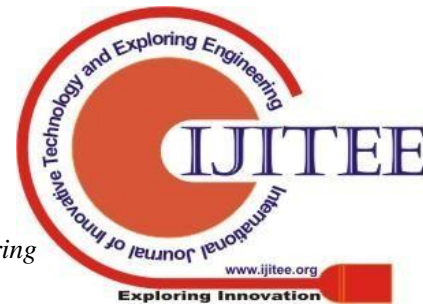


Hyper spectral image classification using Multi Labelled, Multi-Scale and Multi-Angle CNN with MS-MA BT Algorithm

resolution and fusion. For fair contrast, the test samples, the training samples and the parameters in the comparative strategy are the same with our strategy.

Table III shows the convolution layer details in terms of filter size, no., of parameters and no., of filters. Padding and stride are the parameter of convolution layer. Table IV, V, VI gives comparison of angle, resolution and fusion. We can easily conclude that, the accuracy of fusion is higher compared to accuracy of angle and fusion, because it takes in to account both resolution and angle parameter which an important aspect of SAR images.

Table III: Convolution layer details

\begin{tabular}{|c|c|l|}
\hline Filter size & No. of filter & Parameter \\
\hline 3 & 8 & Padding \\
\hline 2 & 2 & Stride \\
\hline 3 & 16 & Padding \\
\hline 2 & 2 & Stride \\
\hline 3 & 32 & Padding \\
\hline
\end{tabular}

Table IV: MS-MA BT multi-scale multi-angle CNN by angle

\begin{tabular}{|c|c|c|c|}
\hline Angle & Accuracy & $\begin{array}{l}\text { GPU Time } \\
\text { in sec }\end{array}$ & $\begin{array}{l}\text { Non-GPU } \\
\text { Time in sec }\end{array}$ \\
\hline $0^{*}$ & $82.57 \%$ & 18 & 36 \\
\hline $90^{*}$ & $84.71 \%$ & 16 & 37 \\
\hline $180^{*}$ & $82.95 \%$ & 22 & 38 \\
\hline $270^{*}$ & $82.98 \%$ & 16 & 36 \\
\hline
\end{tabular}

Table IV represent accuracy, GPU time in sec and NonGPU time in sec. In which 90॰ angle gives higher accuracy than other angles. It takes $16 \mathrm{sec}$ time, whereas $180 \circ$ and $270 \circ$ angle gives around same accuracy but it takes different time. Non-GPU takes more time than GPU in angle, resolution and fusion as well.

Table V: MS-MA BT multi scale multi angle CNN by resolution

\begin{tabular}{|c|l|c|c|}
\hline Resolution & $\begin{array}{l}\text { Accurac } \\
\mathbf{y}\end{array}$ & $\begin{array}{l}\text { GPU Time } \\
\text { in sec }\end{array}$ & $\begin{array}{l}\text { Non-GPU } \\
\text { Time in sec }\end{array}$ \\
\hline $64 \times 64$ & $84.14 \%$ & 17 & 36 \\
\hline $256 \times 256$ & $83.12 \%$ & 17 & 36 \\
\hline $512 \times 512$ & $81.00 \%$ & 13 & 35 \\
\hline
\end{tabular}

Table V shows the MS-MA BT CNN by resolution. As we can see that resolution $64 \times 64$ and $256 \times 256$ take same time but gives different accuracy, where accuracy of $64 \times 64$ is around $84 \% .512 \times 512$ resolution take $13 \mathrm{sec}$ and it gives $81.00 \%$ accuracy.

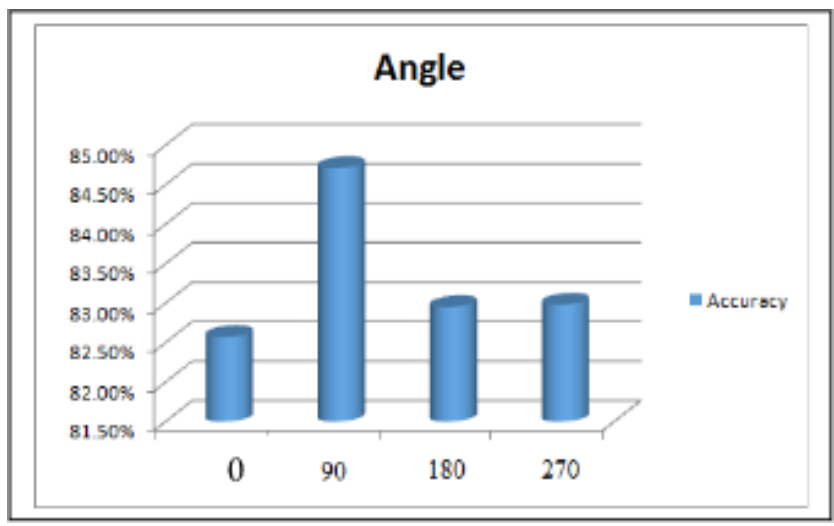

Graph 1: Accuracy of MA CNN with angle variant dataset
a) $0^{2}$
b) $90^{2}$
c) $180^{\circ}$
d) $270^{\circ}$

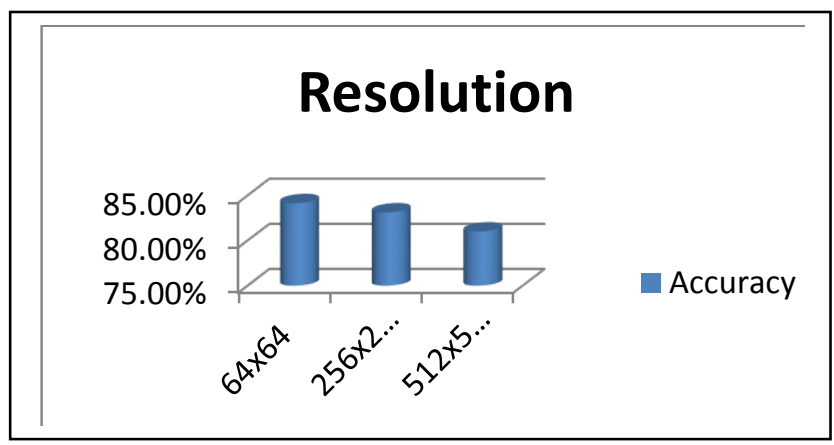

Graph 2: Accuracy of MS CNN with resolution variant dataset
a) $64 \times 64$
b) $256 \times 256$
c) $512 \times 512$

Table VI: Fusion of angle and resolution

\begin{tabular}{|c|c|c|c|}
\hline $\begin{array}{l}\text { Fusion of Angle } \\
\text { and Resolution }\end{array}$ & Accuray & $\begin{array}{l}\text { GPU } \\
\text { Time in } \\
\text { sec }\end{array}$ & $\begin{array}{l}\text { Non-GPU } \\
\text { Time in } \\
\text { sec }\end{array}$ \\
\hline $90^{2}-64 \times 64$ & $84.49 \%$ & 15 & 35 \\
\hline $90^{2}-256 \times 256$ & $85.73 \%$ & 14 & 36 \\
\hline $90^{2}-512 \times 512$ & $87.04 \%$ & 14 & 36 \\
\hline $180^{2}-64 \times 64$ & $84.55 \%$ & 15 & 35 \\
\hline $180^{2}-256 \times 256$ & $88.99 \%$ & 19 & 38 \\
\hline $180^{\circ}-512 \times 512$ & $87.27 \%$ & 15 & 35 \\
\hline $270^{2}-64 \times 64$ & $85.02 \%$ & 16 & 36 \\
\hline $270^{\circ}-256 \times 256$ & $86.14 \%$ & 16 & 36 \\
\hline $270^{\circ}-512 \times 512$ & $88.23 \%$ & 15 & 35 \\
\hline
\end{tabular}

Above table shows fusion of classification \& resolution. In this table we show the classification accuracy, GPU time and Non-GPU time factor of angle and resolution. In fusion table we can clearly see that, it improves the classification accuracy compared to resolution and angle taken individually.

Graph 2 shows the classification accuracy of MS CNN with resolution variant dataset. In this graph $64 \times 64$ has higher accuracy than other resolutions. 


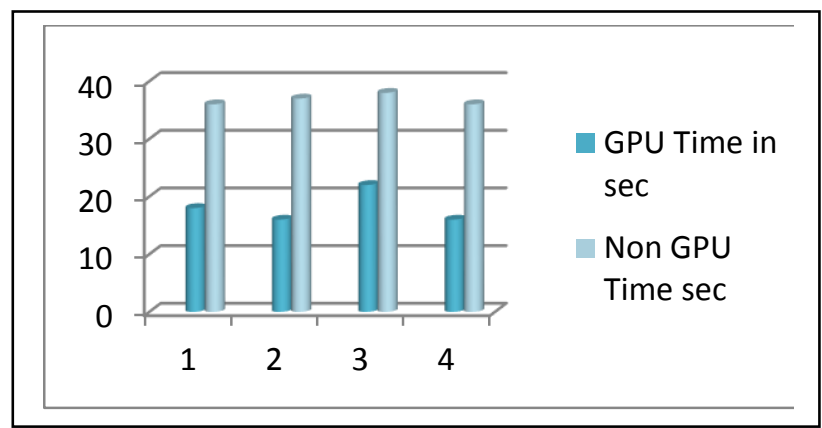

Graph 3: Time consumption by GPU and Non-GPU with angle variant dataset

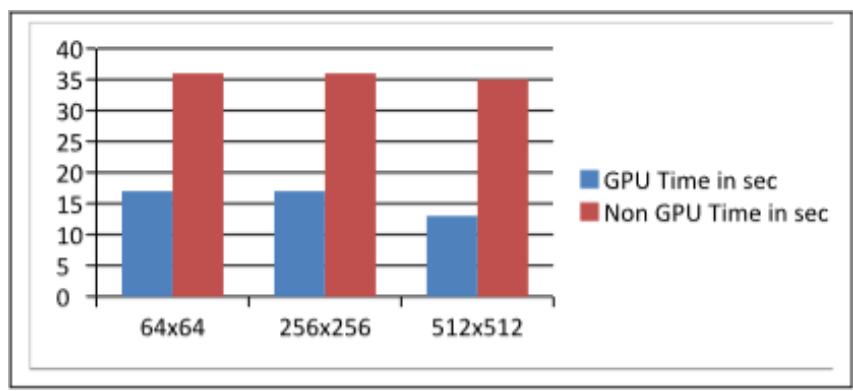

Graph 4: Time consumption by GPU and Non-GPU with resolution variant dataset

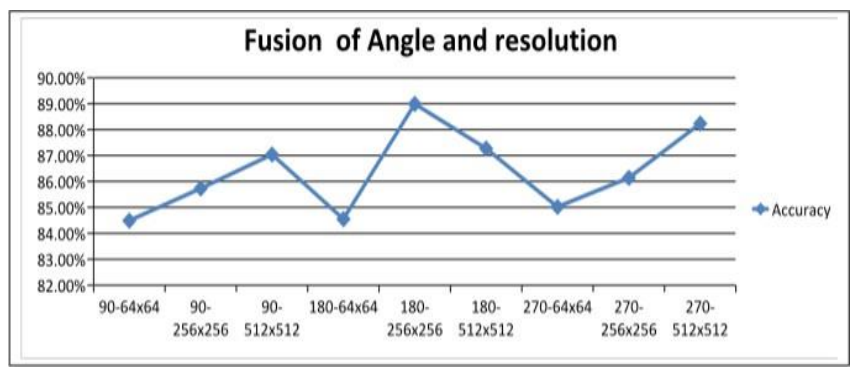

Graph 5: Classification Accuracy of MS-MA CNN on Fused dataset of Angle variant and Resolution variant

Graph 3 shows, time consumption by GPU and Non-GPU with angle variant dataset. In this time required by Non-GPU is more as compared to GPU.

Graph 4 shows, time consumption by GPU and Non-GPU with resolution variant dataset. In this graph time taken for estimating the classification accuracy increases as resolution of the image increases.

Graph 5 represents accuracy of MS-MA CNN on fusion dataset of angle and resolution variant. In this graph we evaluate total fusion accuracy. We found $80^{\circ}-256 \times 256$ has the highest accuracy compared to other fused datasets.

Graph 6 represents time consumption by GPU and Non-GPU with fused dataset. In this graph time taken for estimating the classification accuracy is around same for resolution and angle variation.

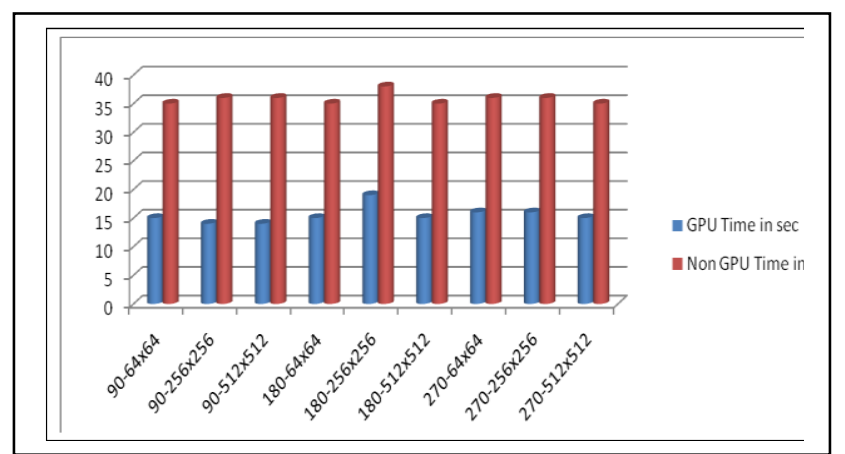

Table VII: Average of Accuracy, GPU time and Non-GPU time analysis with respect to angle, resolution and fusion

\begin{tabular}{|c|c|c|c|}
\hline & $\begin{array}{c}\text { Accurac } \\
\mathbf{y}\end{array}$ & $\begin{array}{c}\text { GPU } \\
\text { time }\end{array}$ & $\begin{array}{c}\text { Non- } \\
\text { GPU } \\
\text { time }\end{array}$ \\
\hline Angle & $83.30 \%$ & 18 & 36.75 \\
\hline $\begin{array}{c}\text { Resoluti } \\
\text { on }\end{array}$ & $82.75 \%$ & 15.66 & 35.67 \\
\hline Fusion & $86.38 \%$ & 15.44 & 35.77 \\
\hline
\end{tabular}

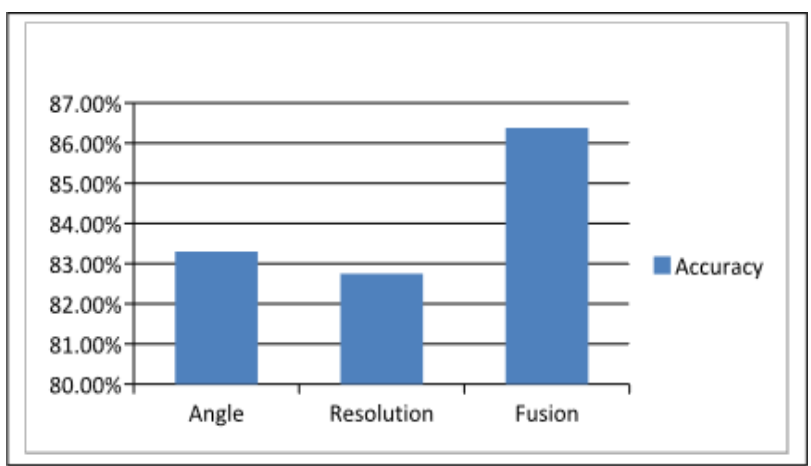

Graph 7: Average of accuracy analysis with respect to angle, resolution and fusion

Graph 7 represent average of accuracy analysis with respect to angle, resolution and fusion. In this graph we found that average of fusion accuracy increases as compared to angle and resolution.

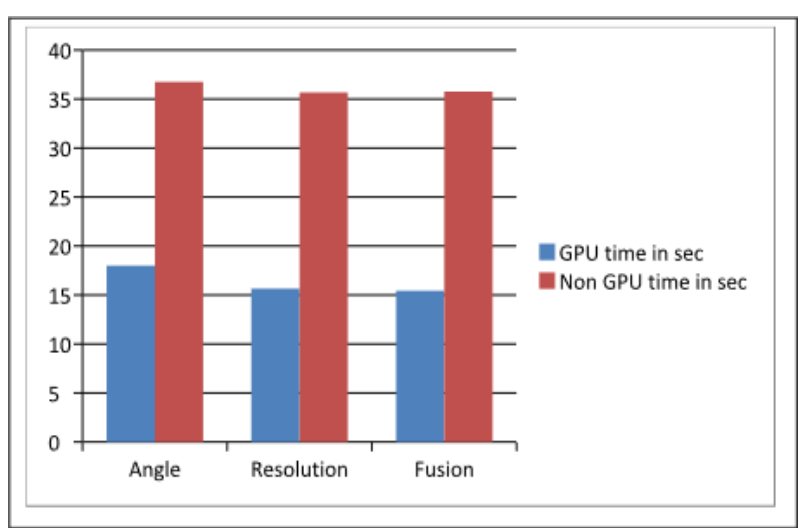

Graph 8: Average of GPU time and Non-GPU time analysis with respect to angle, resolution and fusion

Graph 8 represent GPU time and Non-GPU time analysis

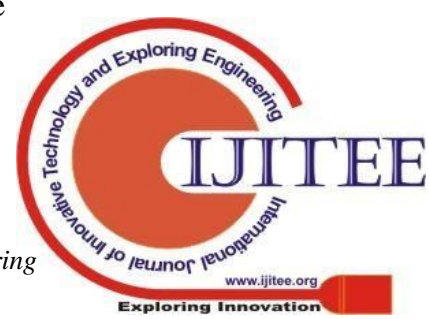




\section{Hyper spectral image classification using Multi Labelled, Multi-Scale and Multi-Angle CNN with MS-MA BT}

Algorithm

with respect to angle, resolution and fusion. In this graph we conclude that GPU time in sec decreases as compared to Non-GPU Time in sec.

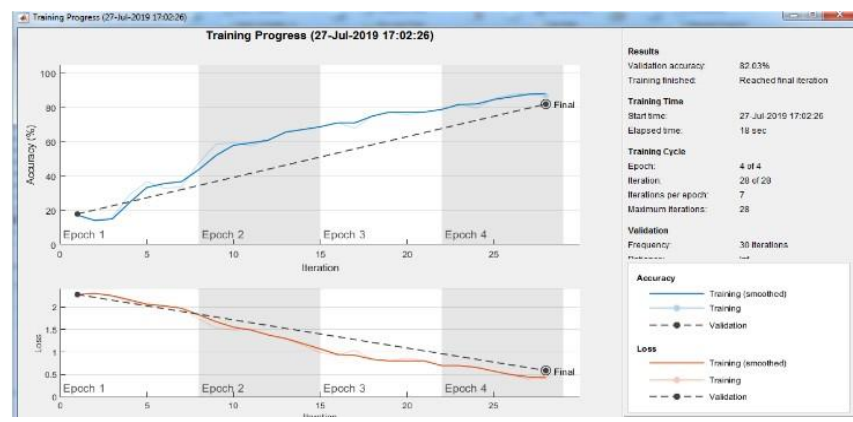

Graph 9: Accuracy and Loss obtained by MS-MA CNN on our Dataset in Matlab ANN Toolbox

In graph 9 we can clearly see that, the classification accuracy comes out to be $82.03 \%$ with 18 secs required for training data on GPU, as the classification accuracy goes on increasing, loss function goes on decreasing.

\section{CONCLUSION}

Our above work represents detailed analysis of hyper spectral SAR image classification using various algorithms. Based on above results it is clear that our newly proposed MS-MA BT CNN algorithm is successfully implemented and tested with highest accuracy of $88.99 \%$ as compared to other traditional algorithms. The results improve to a larger extent when along with the resolution, angle is also taken into consideration. Therefore, our algorithm can be also tested for all kind of image datasets available on Internet as well as real-time datasets of satellite or any other media.

In our implementation it is noticed that NVIDIA GPU played a vital role to reduce time complexity compared to regular CPU Processor. Hence it is recommended to use GPU for higher end Image processing Task.

In future the number of training samples and testing samples can be varied. We will try to implement few more feature extraction technique in fusion with our existing work so as it will serve as comprehensive outcome of our research in the domain.

\section{ACKNOWLEDGMENT}

This work is supported in part by NVIDIA GPU grant program. We thank NVIDIA for giving us Titan XP GPU as a grant to carryout our work in deep learning. We also thank the anonymous reviewers for their insightful comments.

\section{REFERENCES}

[1] Sifan Wu, Junping Zhang, Chunyu Shi, Weike Li, "multiscale spectral-spatialhyperspectralimage classification with adaptive filtering “, IEEE, PP:2591 - 2594, 2018.

[2] Lin He , Jun Li ,Antonio Plaza ,Yuanqing Li ,"Discriminative LowRank Gabor Filtering for Spectral-Spatial Hyperspectral Image Classification”, IEEE,PP:1381 - 1395, Volume:55, 2017.

[3] ShizhiZhao, WeiLi QianDu "Hyperspectral Classification Based on Siamese Neural Network Using Spectral-Spatial Feature ”, IEEE,PP : 2567- 2570,2017.
[4] LichaoMou,PedramGhamisi,XiaoXiangZhu,'Fully convdeconvnetwork for unsupervised spectralspatial feature extraction of hyperspectral imagery via residual learning”, IEEE,PP: 5181-5184, 2017

[5] ShrishBajpa, Harsh Vikram Singh, Naimur Rahman Kidwai, "Feature Extraction \& Classification of Hyperspectral Images using Singular Spectrum Analysis \& Multinomial Logistic Regression Classifiers", IEEE, PP: 97-100, 2018

[6] Miaomiao Liang, Licheng Jiao, Shuyuan Yang, Fang Liu , Biao Hou ,HuanChen,'Deep Multiscale SpectralSpatial Feature Fusion for HyperspectralImage Classification"IEEE, PP : 2911 - 2924,Volume: 11,2018

[7] Mercedes E. Paoletti, Ruben Fernandez-Beltran, "Deep Pyramidal Residual Networks for Spectral-Spatial Hyperspectral Image Classification", IEEE, PP: 740 - 754, Volume: 57,2018

[8] Atif Mughees, Linmi Tao," hyper-pixel based deep learning for hyperspectral image classification”, IEEE, PP:840-844, 2017

[9] Atif Mughees, Ahmad Ali, Linmi Tao, "Hyperspectral image classification via shape-adaptive deep learning”, IEEE,PP:375-379,2017

[10] Shakati Sharama, rishan Mohan Bhuddhi, Raju, Gaurav Kumarda Shondhi, "Hyper spectrum image classification using colony optimization algorithm based on joint spectrum special parameters", IEEE, PP:3210-3213, 2017

[11] Wei Fu ,Shutao Li , Leyuan Fang ,Jón Atli Benediktsson, ”Spectralspatial online dictionary learning for hyperspectral image classification”, IEEE, PP:3724-3727, 2017

[12] SenJia, XiupingJia "Spectral-Spatial Gabor Surface Feature Fusion Approach for Hyperspectral Imagery Classification”, IEEE, PP: 1142 1154,2018

[13] Nanjun He, Mercedes E. Paoletti , Juan Mario Haut , Leyuan Fang ,Shutao Li ,Antonio Plaza , Javier Plaza "Feature ExtractionWith Multiscale Covariance Maps for Hyperspectral Image Classification”, IEEE, Volume:57, PP: 755 - 769,2018

[14] Lin He, Jun Li, Chenying Liu et al., "Recent Advances on SpectralSpatial Hyperspectral Image Classification: An Overview and New Guidelines" IEEE, Volume: 56, PP: 1579 - 1597, 2018

[15] Andreia Valentina Miclea, Romulus Terebes, Ioana Ilea, Monica Borda, "Hyperspectral Image Classification using Combined Spectral-Spatial Denoising and Deep Learning Techniques", IEEE,2018

[16] Simranjit Singh, Singara Singh Kasana, "Spectral-Spatial Hyperspectral Image Classification using Deep Learning”, IEEE,PP:411-417,2017

[17] Cheng Deng, Yumeng Xue ; Xianglong Liu ; Chao Li ; Dacheng Tao "Active Transfer Learning Network: A Unified Deep Joint SpectralSpatial Feature Learning Model for Hyperspectral Image Classification", PP: 1741 - 1754,Volume:57, 2018

[18] Yuemei Ren, Liang Liao, Yanning Zhang, "Hyperspectral Image Spectral-Spatial Feature Extraction via Tensor Principal Component Analysis", Volume:14, PP:1431 - 1435,2017

[19] WEN SHU et al "Hyperspectral Image Classification Using SpectralSpatial Features with Informative Samples", IEEE, PP. 20869 $-20878,2019$

[20] YipingDuan et al, "Multi-scale Convolutional Neural Network for SAR Image Semantic Segmentation”, IEEE, 2018

[21] Yanhui Guo, Han Cao, Jianjun Bai and Yu Bai, "High Efficient Deep Feature Extraction and Classification of Spectral-Spatial Hyperspectral Image Using Cross Domain Convolution Neural Networks" IEEE, vol no: 12 , PP: $345-356,2019$ 